\title{
Measurement of Energy Efficiency Metrics of Data Centers. Case Study: Higher Education Institution of Barranquilla
}

DOI

https://doi.org/10.1007/978-3-030-63319-6 3

\section{Autores}

Leonel Hernandez, Hugo Hernandez, Mario Orozco, Gabriel Piñeres, Jesus GarciaGuiliany

\begin{abstract}
Data centers have become fundamental pillars of the network infrastructures of the various companies or entities regardless of their size. Since they support the processing, analysis, assurance of the data generated in the network, and by the applications in the cloud, which every day increases its volume thanks to diverse and sophisticated technologies. The management and storage of this large volume of information make the data centers consume a lot of energy, generating great concern to owners and administrators. Green Data Center (GDC) is a solution for this problem, reducing the impact produced by the data centers in the environment through the monitoring and control of these and to the application of standards-based on metrics. Although each data center has its particularities and requirements, the metrics are the tools that allow us to measure the energy efficiency of the data center and evaluate if it is friendly to the environment (1.Adv. Intell. Syst.

Comput. 574:329-340). The objective of the study is to calculate these metrics in the data centers of a Higher Education Institution in Barranquilla, on both campuses, and the analysis of these will be carried out. It is planned to extend this study by reviewing several metrics to conclude, which is the most efficient and which allows defining the guidelines to update or convert the data center in a friendly environment. The research methodology used for the development of the project is descriptive and no-experimental.
\end{abstract}

\section{Palabras clave}

Energy, Green data centers, Performance metrics, Environment 